\title{
EMCDDA publishes guidelines on testing for HIV, viral hepatitis and other infections in injecting drug users
}

L Wiessing (Lucas.Wiessing@emcdda.europa.eu)'1, H Blystad (hans.blystad@fhi.no)2

1. European Monitoring Centre for Drugs and Drug Addiction (EMCDDA), Lisbon, Portugal

2. Norwegian Institute of Public Health, Oslo, Norway

Citation style for this article:

Wiessing L, Blystad H. EMCDDA publishes guidelines on testing for HIV, viral hepatitis and other infections in injecting drug users. Euro Surveill. 2010;15(48): pii=19735. Available online: http://www.eurosurveillance.org/ViewArticle. aspx?Articleld=19735

Article published on 2 December 2010

On 1 December 2010, World AIDS Day, the European Monitoring Centre for Drugs and Drug Addiction (EMCDDA) published guidelines on testing for HIV, viral hepatitis and other infectious diseases in injecting drug users (IDU) [1]. The new guidelines recommend a strategy to increase the uptake of testing for HIV and other infections among IDU in Europe and beyond. This would enable earlier treatment of infected individuals which lowers the risk of further spread.

Provided testing is carried out with informed consent, with pre- and post-testing counselling and the confidentiality of test results can be guaranteed, the guidelines recommend that IDU should be offered the following tests regularly (depending on infection risks this can be up to once or even twice per year):

- serology tests for HIV, hepatitis B, hepatitis C, hepatitis $D$ (if there is evidence of chronic or recent hepatitis B), hepatitis A and syphilis;

- $\quad$ swab for culture from abscesses and skin lesions;

- biochemical tests (to determine levels of alanine aminotransferase (ALAT), aspartate aminotransferase (ASAT) and bilirubin);

- other general blood tests (to determine erythrocyte sedimentation rate (ESR), levels of C-reactive protein (CRP) and haemoglobin, and white blood cell count);

- tests for tuberculosis.

The uptake of testing for HIV and other infectious diseases among IDU is still often low in Europe (EMCDDA unpublished data). IDU remain a relatively 'hidden' population, as they are often not regularly in contact with health services, and their infections may remain unnoticed for many years. Infectious diseases are among the most serious health consequences of injecting drug use and can lead to substantial healthcare costs. IDU are vulnerable to a range of infectious diseases through a variety of risky behaviours, such as needle sharing and sex work, and because of circumstances such as poor hygiene, homelessness and poverty.
The guidelines are accompanied by a recommended package of prevention (including vaccination), primary care and referral routines in relation to IDU and infectious diseases. They were developed in collaboration with a range of experts and have been distributed to professionals throughout the European Union and worldwide.

References

1. European Monitoring Centre for Drugs and Drug Addiction (EMCDDA). Guidelines for testing HIV, viral hepatitis and other infections in injecting drug users. Lisbon: EMCDDA; 2010. Available from: http://www.emcdda.europa.eu/publications/ manuals/testing-guidelines 